\title{
An Empirical Evaluation of LFG-DOP
}

\author{
Rens Bod \\ Informatics Research Institute, University of Leeds, Leeds LS2 9JT, \& \\ Institute for Logic, Language and Computation, University of Amsterdam \\ rens@scs.leeds.ac.uk
}

\begin{abstract}
This paper presents an empirical assessment of the LFGDOP model introduced by Bod \& Kaplan (1998). The parser we describe uses fragments from LFG-annotated sentences to parse new sentences and Monte Carlo techniques to compute the most probable parse. While our main goal is to test Bod \& Kaplan's model, we will also test a version of LFG-DOP which treats generalized fragments as previously unseen events. Experiments with the Verbmobil and Homecentre corpora show that our version of LFG-DOP outperforms Bod \& Kaplan's model, and that LFG's functional information improves the parse accuracy of tree structures.
\end{abstract}

\section{Introduction}

We present an empirical evaluation of the LFG-DOP model introduced by Bod \& Kaplan (1998). LFG-DOP is a Data-Oriented Parsing (DOP) model (Bod 1993, 98) based on the syntactic representations of LexicalFunctional Grammar (Kaplan \& Bresnan 1982). A DOP model provides linguistic representations for an unlimited set of sentences by generalizing from a given corpus of annotated exemplars. It operates by decomposing the given representations into (arbitrarily large) fragments and recomposing those pieces to analyze new sentences. The occurrence-frequencies of the fragments are used to determine the most probable analysis of a sentence.

So far, DOP models have been implemented for phrase-structure trees and logical-semantic representations (ct. Bod 1993, 98; Sima'an 1995, 99; Bonnema et al. 1997; Goodman 1998). However, these DOP models are limited in that they cannot account for underlying syntactic and semantic dependencies that are not reflected directly in a surface tree. DOP models for a number of richer representations have been explored (van den Berg et al. 1994; Tugwell 1995), but these approaches have remained context-free in their generative power. In contrast, Lexical-Functional Grammar (Kaplan \& Bresnan 1982) is known to be beyond context-free. In Bod \& Kaplan (1998), a first DOP model was proposed based on representations defined by LFG theory ("LFG-DOP"). 1 This model was

\footnotetext{
1 DOP models have recently also been proposed for TreeAdjoining Grammar and Head-driven Phrase Structure Grammar (cf. Neumann \& Flickinger 1999).
}

studied from a mathematical perspective by Cormons (1999) who also accomplished a first simple experiment with LFG-DOP. Next, Way (1999) studied LFG-DOP as an architecture for machine translation. The current paper contains the first extensive empirical evaluation of LFG-DOP on the currently available LFG-annotated corpora: the Verbmobil corpus and the Homecentre corpus. Both corpora were annotated at Xerox PARC.

Our parser uses fragments from LFG-annotated sentences to parse new sentences, and Monte Carlo techniques to compute the most probable parse. Although our main goal is to test Bod \& Kaplan's LFGDOP model, we will also test a modified version of LFG-DOP which uses a different model for computing fragment probabilities. While Bod \& Kaplan treat all fragments probabilistically equal regardless whether they contain generalized features, we will propose a more fine-grained probability model which treats fragments with generalized features as previously unseen events and assigns probabilities to these fragments by means of discounting. The experiments indicate that our probability model outperforms Bod \& Kaplan's probability model on the Verbmobil and Homecentre corpora.

The rest of this paper is organized as follows: we first summarize the LFG-DOP model and go into our proposed extension. Next, we explain the Monte Carlo parsing technique for estimating the most probable LFGparse of a sentence. In section 3, we test our parser on sentences from the LFG-annotated corpora.

\section{Summary of LFG-DOP and an Extension}

In accordance with Bod (1998), a particular DOP model is described by specifying settings for the following four parameters:

- a formal definition of a well-formed representation for utterance analyses,

- a set of decomposition operations that divide a given utterance analysis into a set of fragments,

- a set of composition operations by which such fragments may be recombined to derive an analysis of a new utterance, and

- a probability model that indicates how the probability of a new utterance analysis is computed. 
In defining a DOP model for Iexical-Functional Grammar representations, Bod \& Kaplan (1998) give the following settings for DOP's four parameters.

\subsection{Representations}

The representations used by LFG-DOP are directly taken from LFG: they consist of a c-structure, an f-structure and a mapping $\phi$ between them (see Kaplan \& Bresnan 1982). The following figure shows an example representation for the utterance Kim eats. (We leave out some features to keep the example simple.)

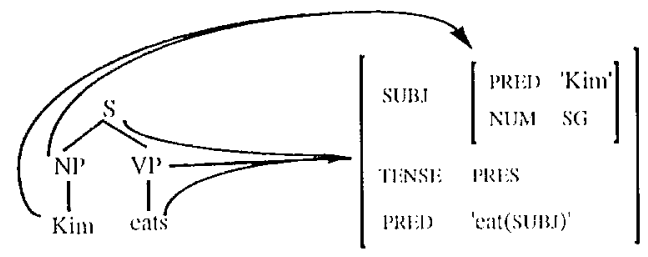

Fïgure 1. A representation for Kim cats

Bod \& Kaplan also introduce the notion of acesssibility which they later use for defining the decomposition operations of LFG-DOP:

An f-structure unit $f$ is $\phi$-acessible from a node $n$ iff either $n$ is $\phi$-linked to $f$ (that is, $f=\phi(n))$ or $f$ is contained within $\phi(n)$ (that is, there is a chatin of attributes that leads from $\phi(n)$ to $f)$.

According to the IJG representation theory, e-structures and f-structures must satisly certain formal wellformedness conditions. A c-structure/l-structure pair is a valid $\mathrm{LFG}$ representation only if it satisfies the Nonbranching Dominance, Uniqueness, Coherence and Completeness conditions (sec Kaplan \& Bresnan 1982).

\subsection{Decomposition operations and Fragments}

The ragments for LIG-DOP consist of connected subtrees whose nodes are in p-correspondence with the correponding sub-units of f-structures. 'lo give a precise definition of LliG-DOP' fragments, it is convenient to recall the decomposition operations employed by the simpler "Tree-DOP" model which is based on phrasestructure trees only (Bod 1998):

(1) Root: the Root operation selects any node of a tree to be the root of the new subtree and crases all nodes except the selected node and the nodes it dominates.

(2) Frontier: the Frontier operation then chooses a set (possibly empty) of nodes in the new subtree different from its root and erases all subtrees dominated by the chosen nodes.

Bod \& Kaplan extend Tree-DOP's Root and Frontie. operations so that they also apply to the nodes of the e- structure in $\mathrm{LFG}$, while respecting the fundamental principles of e-structure/f-structure correspondence.

When a node is selected by the Root operation, all nodes outside of that node's subtre are eased, just as in Tree-DOP. Further, for LlG-DOP, all $\phi$ links leaving the erased nodes are removed and all i-structure units that are not $\phi$-accessible from the remaining nodes are erased. For example, if Root selects the NP in figure 1 , then the f-structure corresponding to the $S$ node is erased, giving ligure 2 as a possible fragment:

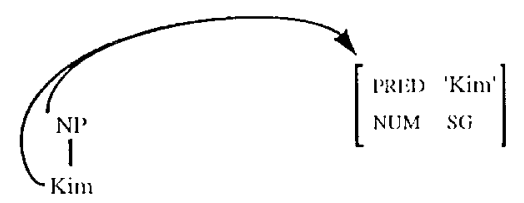

liggure 2. An LFG-IOOP fragment oblained by Root

In addition the Root operation deletes from the remaining $f$-structure all semantic forms that are local to f-structures that correspond to erased c-structure nodes, and it thereby also maintains the fundamental (wo-way connection between words and meanings. Thus, if Root selects the VP node so that the NP is erased, the subject semantic form "Kim" is also deleted:

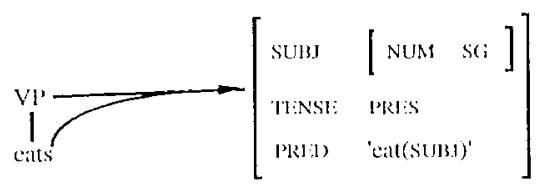

ligure 3. Another LJGG-IDOP' fragment

As with 'Tree-DOP, the Frontier operation then selects a set of frontier nodes and deletes all subtrees they dominate. Like Root, it also removes the $\phi$ links of the deleted nodes and erases any semantic form that corresponds to any of those nodes. Frontier does not delete any other i-structure leatures, however. For instance, if the NP in ligure $I$ is selected as a frontic node, frontier crases the predicate "Kim" from the frigment:

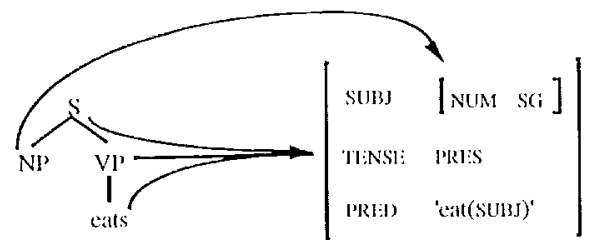

Figure 4. A fronticr-gencrated fragment

Finally, Bod \& Kaplan present a third decomposition operation, Discard, defined to construct generalizations of the fragments supplicd by Root and Frontier. Discard acts to delete combinations of attribute-value pairs subject to the following condition: Discard does not delete paits whose values $\phi$-correspond to remaining $\mathrm{c}$ - 
structure nodes. Discard produces fragments such as in figure 5, where the subject's number in figure 3 has been deleted:

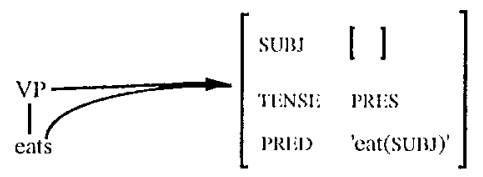

Figure 5. A Discard-generated fragment

\subsection{The composition operation}

In LFG-DOP the operation for combining fragments, indicated by ${ }^{\circ}$, is carried out in two steps. First the cstructures are combined by left-most substitution subject to the category-matching condition, just as in Tree-DOP (cf. Bod 1993, 98). This is followed by the recursive unification of the f-structures corresponding to the matching nodes. A derivation for an LFG-DOP representation $R$ is a sequence of fragments the first of which is labeled with $S$ and for which the iterative application of the composition operation produces $R$.

The two-stage composition operation is illustrated by a simple example. We therefore assume a corpus containing the representation in figure 1 for the sentence Kim eats and the representation in figure 6 for the sentence John fell.

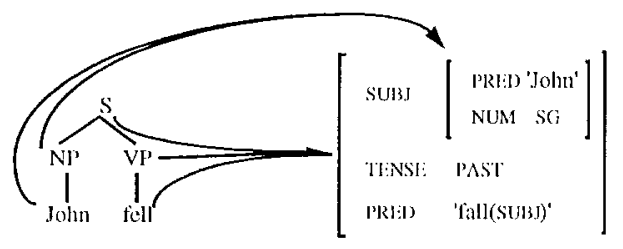

Figure 6. Corpus representation for John fell

Figure 7 shows the effect of the LFG-DOP composition operation using two fragments from this corpus, resulting in a representation for the new sentence Kim fell.

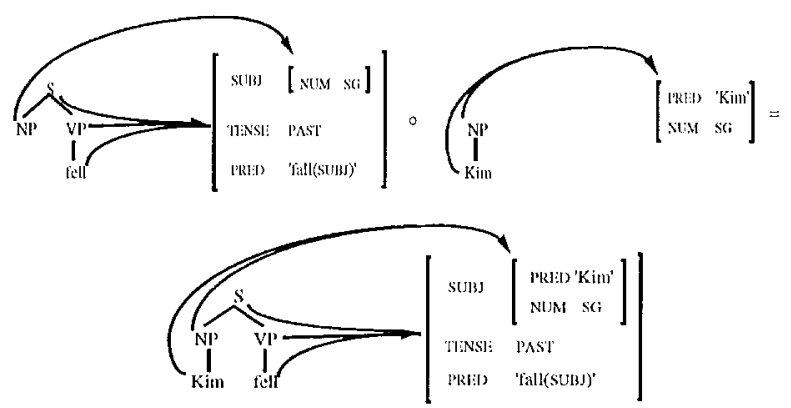

Figure 7. Illustration of the composition operation

This representation satisfies the well-formedness conditions and is therefore valid. Note that the sentence Kim fell can be parsed by fragments that are generated by the decomposition operations Root and Frontier only, without using generalized fragments (i.e. fragments generated by the Discard operation). Bod \& Kaplan (1998) call a sentence "grammatical with respect to a corpus" if it can be parsed without generalized fragments. Generalized fragments are needed only to parse sentences that are "ungrammatical with respect to the corpus".

\subsection{Probability models}

As in Tree-DOP, an LFG-DOP representation $R$ can typically be derived in many different ways. If each derivation $D$ has a probability $\mathrm{P}(D)$, then the probability of deriving $R$ is the sum of the individual derivation probabilities, as shown in (1):

(1) $\mathrm{P}(R)=\Sigma_{D \text { derives } R} \mathrm{P}(D)$

An LFG-DOP derivation is produced by a stochastic process which starts by randomly choosing a fragment whose c-structure is labeled with the initial category (e.g. S). At each subsequent step, a next fragment is chosen at random from among the fragments that can be composed with the current subanalysis. The chosen fragment is composed with the current subanalysis 10 produce a new one; the process stops when an analysis results with no non-terminal leaves. We will call the set of composable fragments at a certain step in the stochastic process the competition set at that step. Let $\mathrm{CP}(f \mid \mathrm{CS})$ denote the probability of choosing a fragment $f$ from a competition set $\mathrm{CS}$ containing $f$, then the probability of a derivation $D=\left\langle f_{1}, f_{2} \ldots f_{k}\right\rangle$ is

$$
\mathrm{P}\left(<f_{1}, f_{2} \ldots f_{k}>\right)=\prod_{i} \mathrm{CP}\left(f_{i} \mid \mathrm{CS}_{i}\right)
$$

where the competition probability $\mathrm{CP}(f \mid \mathrm{CS})$ is expressed in terms of fragment probabilities $P(f)$ :

$$
\mathrm{CP}(f \mid \mathrm{CS})=\frac{\mathrm{P}(f)}{\sum_{f^{\prime} \in \mathrm{CS}} \mathrm{P}\left(f^{\prime}\right)}
$$

Bod \& Kaplan give three definitions of increasing complexity for the competition set: the first definition groups all fragments that only satisfy the Categorymatching condition of the composition operation (thus leaving out the Uniqueness, Coherence and Completeness conditions); the second definition groups all fragments which satisly both Category-matching and Uniqueness; and the third definition groups all fragments which satisfy Category-matching, Uniqueness and Coherence. Bod \& Kaplan point out that the Completeness condition cannot be enforced at each step of the stochastic derivation process. It is a property of the final representation which can only be enfored by sampling valid representations from the output of the stochastic process.

In this paper, we will only deal with the third definition of competition set, as it selects only those 
fragments at each derivation step that may finally result in a valid LFG representation, thus reducing the off-line validity checking just to the Completeness condition.

Notice that the computation of the competition probability in (3) still requires a definition for the fragment probability $\mathrm{P}(f)$. Bod \& Kaplan define the probability of a fragment simply as its relative frequency in the bag of all fragments generated from the corpus. Thus Bod \& Kaplan do not distinguish between Root/Frontier-generated fragments and Discardgenerated lragments, the latter being generalizations over Root/Frontier-generated lragments. Although Bod \& Kaplan illustrate with a simple example that their probability model exhibits a preference for the most specific representation containing the fewest feature generalizations (mainly because specific representations tend to have more derivations than generalized representations), they do not perform an empirical cvaluation of their model. We will assess their model on the LFG-annotated Verbmobil and Homecentre corpora in section 3 of this paper.

However, we will also assess an alternative definition of fragment probability which is a refinement of Bod \& Kaplan's model. This definition does distinguish between fragments supplied by Root/Frontier and fragments supplied by Discard. We will treat the first type of fragments as seen events, and the second type of fragments as previously unseen events. We thus create two separate bags corresponding to two separate distributions: a bag with fragments generated by Root and Frontier, and a bag with fragments generated by Discard. We assign probability mass to the fragments of each bag by means of discounting: the relative frequencies of seen events are discounted and the gained probability mass is reserved for the bag of unsecn events (ci. Ney et al. 1997). We aceomplish this by a very simple estimator: the Turing-Good estimator (Good 1953) which computes the probability mass of unseen events as $n_{1} / N$ where $n_{1}$ is the number of singleton events and $N$ is the total number of seen events. This probability mass is assigned to the bag of Discardgenerated fragments. The remaining mass $\left(1-n_{1} / N\right)$ is assigned to the bag of Rootfrontier-generated fragments. Thus the total probability mass is redistributed over the seen and unseen fragments. The probability of each fragment is then computed as its relative frequency ${ }^{2}$ in its bag multiplied by the probability mass assigned to this bag. Iet $|f|$ denote the frequency of a fragment $f$, then its probability is given by:

\footnotetext{
2 Bod (2000) discusses some alternative fragment probability estimators, e.g. based on maximum likelihood.
}

$$
\mathrm{P}(f \mid f \text { is generated by Root/Fronticr })=
$$

$$
\left(1-n_{\mathrm{l}} / N\right) \frac{|f|}{\sum_{f: f \text { is generated by Rool/Frontier }|f|} \mid}
$$

$$
\mathrm{P}(f \mid f \text { is generated by Discard })=
$$

$$
\left(n_{1} / N\right) \frac{|f|}{\sum_{f^{\prime}: f^{\prime} \text { is generited by Discard }\left|f^{\prime}\right|}}
$$

Note that this probability model assigns less probability mass to Discard-generated fragments than Bod \& Kaplan's model. For each Root/Frontier-generated fragment there are exponentially many Discardgenerated fragments (exponential in the number of features the fragment contains), which means that in Bod \& Kaplan's model the Discard-generated fragments absorb a vast amount of probability mass. Our model, on the other hand, assigns a fixed probability mass to the distribution of Discard-generated fragments and therefore the exponential explosion of these fragments does not affect the probabilities of Roof/Frontiergencrated fragments.

\section{Testing the IJG-DOP model}

\subsection{Computing the most probable analysis}

In his PhD-thesis, Cormons (1999) describes a parsing algorithm for ILFG-DOP which is based on the Tree-DOP parsing technique given in Bod (1998). Cormons first converts LFG-representations into more compact indexed trees: each node in the c-structure is assigned an index which refers to the $\phi$-corresponding f-structure unit. For example, the representation in figure 6 is indexed ats

\section{(S.1 (NP.2 John.2) \\ (VP.1 Iell.1)}

where

$$
\begin{aligned}
1 \rightarrow \perp & (\text { SUBJ }=2) \\
& (\text { TENSE = PAST }) \\
& (\text { PRED }=\text { fall }(\text { SUBJ }))] \\
2 \rightarrow> & (\text { PRED }=\text { John }) \\
& (\text { NUM }=\text { SG })]
\end{aligned}
$$

The indexed trees are then fragmented by applying the Tree-DOP decomposition operations described in section 2. Next, the LFG-DOP decomposition operations Root, Frontier and Discard are applied to the f-structure units that correspond to the indices in the c-structure subtrees. Having obtained the set of LFG-DOP fragments in this way, each test sentence is parsed by a bottom-up chart parser using initially the indexed subtrees only. Thus only the Category-matching condition is enforced during 
the chart-parsing process. The Uniqueness and Coherence conditions of the corresponding f-structure units are enforced during the disambiguation (or chartdecoding) process. Disambiguation is accomplished by computing a large number of random derivations from the chart; this technique is known as "Monte Carlo disambiguation" and has been extensively described in the literature (c.g. Bod 1998; Chappelier \& Rajman 1998; Goodman 1998). Sampling a random derivation from the chart consists of choosing at random one of the fragments from the set of composable fragments at every labeled chart-entry (in a top-down, leftmost order so as to maintain the LFG-DOP derivation order). Thus the competition set of composable fragments is computed on the fly at each derivation step during the Monte Carlo sampling process by grouping the f-structure units that unify and that are coherent with the subderivation built so far.

As mentioned in 2.4, the Completeness condition can only be checked after the derivation process. Inconplete derivations are simply removed from the sampling distribution. After sampling a large number of random derivations that satisfy the LFG validity requirements, the most probable analysis is estimated by the analysis which results most often from the sampled derivations. For our experiments in section 3.2, we used a sample size of $N=10,000$ derivations which corresponds to a maximal standard crror $\sigma$ of $0.005(\sigma \leq 1 /(2 \sqrt{ } N)$, see Bod 1998).

\subsection{Experiments with I.FG-DOP}

We tested LFG-DOP on two LFG-annotated corpora: the Verbmobil corpus, which contains appointment planning dialogues, and the Homecentre corpus, which contains Xerox printer documentation. Both corpora have been annotated by Xerox PARC. They contain packed LFGrepresentations (Maxwell \& Kaplan 1991) of the grammatical parses of each sentence together with an indication which of these parses is the correct one. The parses are represented in a binary form and were debinarized using software provided to us by Xerox PARC. ${ }^{3}$ For our experiments we only used the correct parses of each sentence resulting in 540 Verbmobil parses and 980 Homecentre parses. Each corpus was divided into a $90 \%$ training set and a $10 \%$ test set. This division was random except for one constraint: that all the words in the test set actually occurred in the training set. The sentences from the test set were parsed and disambiguated by means of the fragments from the training set. Due to memory limitations, we limited the depth of the indexed subtrees to 4 . Because of the small

${ }^{3}$ Thanks to Hadar Shemtov for providing us with the relevant soltware. size of the corpora we averaged our results on 10 different training/test set splits. Besides an exact match accuracy metric, we also used a more fine-grained metric based on the well-known PARSEVAL metrics that evaluate phrase-structure trees (Black et al. 1991). The PARSEVAL metrics compare a proposed parse $P$ with the corresponding correct treebank parse $T$ as follows:

$$
\begin{aligned}
& \text { Precision }=\frac{\# \text { correct constituents in } P}{\# \text { constituents in } P} \\
& \text { Recall }=\frac{\# \text { correct constituents in } P}{\# \text { constituents in } T}
\end{aligned}
$$

In order to apply these metrics to LFG analyses, we extend the PARSEVAL notion of "correct constituent" in the following way: a constituent in $P$ is correct if there exists a constituent in $T$ of the same label that spans the same words and that $\phi$-corresponds to the same istructure unit.

We illustrate the evaluation metrics with a simple example. In the next figure, a proposed parse $P$ is compared with the correct parse $T$ for the test sentence Kim fell. The proposed parse is incorrect since it has the incorrect feature value for the TENSE attribute. Thus, if this were the only test sentence, the exact match would be $0 \%$. The precision, on the other hand, is higher than $0 \%$ as it compares the parse on a constituent basis. Both the proposed parse and the correct parse contain three constituents: S, NP and VP. While all three constituents in $P$ have the same label and span the same words as in $T$, only the NP constituent in $P$ also maps to the same fstructure unit as in $T$. The precision is thus equal to $1 / 3$. Note that in this example the recall is equal to the precision, but this need not always be the case.

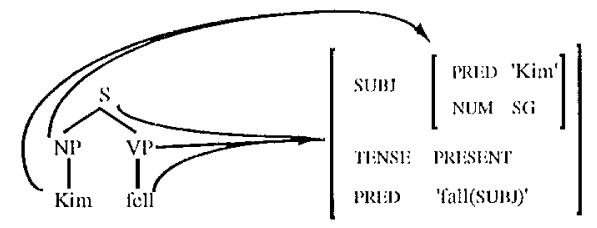

Proposed parse $P$

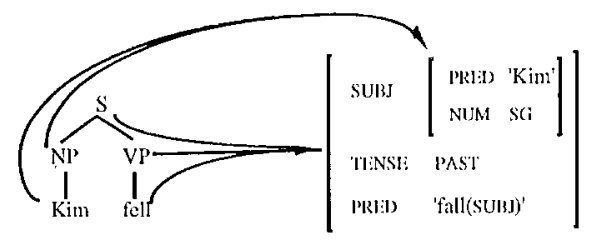

Correct parse $T$

In our experiments we are first of all interested in comparing the performance of Bod \& Kaplan's probability model against our probability model (as explained in section 2.4). Moreover, we also want to 
study the contribution of Discard-generated fragnents 10 the parse accuracy. We therefore created for each training set two sets of fragments: one which contains all fragments (up to (lepth 4) and one which excludes the fragments gencrated by Discard. The exclusion of the Discard-generated fragments means that all probability mass goes to the hagments generated by Root and frontier in which case our model is equivalent 10 Bod \& Kaplan's. The following two tables present the results of our experiments where +Discard refers to the full set of fragments and-Discard refers to the fragment set without Discard-generated fragments.

\begin{tabular}{|c|c|c|c|c|c|c|}
\hline \multirow[b]{2}{*}{ 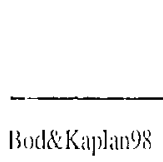 } & \multicolumn{2}{|c|}{$\begin{array}{c}\text { Exact Match } \\
\text { +1)iscard - J)iscard }\end{array}$} & \multicolumn{2}{|c|}{$\begin{array}{c}\text { P'lecision } \\
\text { + Miscard - Discard }\end{array}$} & \multicolumn{2}{|c|}{$\begin{array}{c}\text { Recill } \\
\text { +Discand -Disciat }\end{array}$} \\
\hline & $1.1 \%$ & $35.2 \%$ & $13.8 \%$ & $76.0 \%$ & $11.5 \%$ & $7.1 .9 \%$ \\
\hline OulModel & $35.9 \%$ & $35.2 \%$ & $77.5 \%$ & $76.0 \%$ & $76.1 \%$ & $7+.9 \%$ \\
\hline
\end{tabular}

Table 1. lixperimental results on the Verbmobil corpus

\begin{tabular}{|c|c|c|c|c|c|c|}
\hline & \multicolumn{2}{|c|}{$\begin{array}{c}\text { lixact Match } \\
\text { +Discard - Disciad }\end{array}$} & \multicolumn{2}{|c|}{$\begin{array}{c}\text { Precision } \\
\text { +Discand - l)iscand }\end{array}$} & \multicolumn{2}{|c|}{ 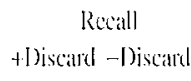 } \\
\hline BodkKaplang8 & $2.7 \%$ & $37.9 \%$ & $17.1 \%$ & $77.8 \%$ & $15.5 \%$ & 77.29 \\
\hline OurModel & $38.4 \%$ & $37.9 \%$ & $80.0 \%$ & $77.8 \%$ & $78.6 \%$ & $77.2 \%$ \\
\hline
\end{tabular}

Table 2. Jixperimental results on the Homecentre corpus

The lables show that Bod \& Kaplants model seores extremely bad if all fragments are used: the exact match is only $1.1 \%$ on the Verbmobil corpus and $2.7 \%$ on the Homecentre corpus, whereas our model scores respectively $35.9 \%$ and $38.4 \%$ on these corpora. $\Lambda \mathrm{Iso}$ the more finc-grained precision and recall scores of Bod \& Kaplan's model are quite low: e.g. $13.8 \%$ and $11.5 \%$ on the Verbmobil corpus, where our model obtains $77.5 \%$ and $76.4 \%$. We found out that even for the few test sentences that occur literally in the training set, Jod \& Kaplan's model does not always generate the correct analysis, whereas our model does. Interestingly, the accuracy of Bod \& Kaplan's model is much higher if Discard-generated fragments are excluded. This suggests that treating generalized fragments probabilistically in the same way as ungeneralized fragments is harmful. Cormons (1999) has made a mathematical observation which also shows that generalized fragments can get too much probability mass.

The tables also show that our way of assigning probabilitics to Discard-generated langments leads only to a slight accuracy increase compared to the experiments in which Discard-generated framents are excluded). According to paired t-testing none of these differenes in accuracy were statistically significant. This suggests that Discard-generated fragments do not significantly contribute to the parse accuracy, or that perhaps these fragments are too mumerous to be reliably estimated on the batsis of our small corpora. We also varied the probability mass assigned to Discardgenerated fragments: except for very small $(\leq 0.01)$ or large values ( $\geq 0.88$ ), which led to an accuracy decrease, there was no signilicant change. ${ }^{4}$

It is difficult to saly how good or bad our results are with respect to other approaches. The only other published results on the LFG-annotated Verbmobil and Homecentre corpora are by Johnson et al. (1999) and Johnson \& Ric/ler (2000) who use a log-linear model to estimate probabilities. But while we first parse the test sentences with fragments from the training set and subsequently compute the most probable parse, Johnson el al. directly use the packed I, IG-representations from the test set to select the most probable parse, thereby completely skipping the parsing phase (Mark Joluson, p.c.). Moreover, $42 \%$ of the Verbmobil sentences and $51 \%$ of the flomecentre sentences are unambiguous (i.e. their packed $\mathrm{L} / \mathrm{l}$ G-representations contain only one analysis), which makes Johnson et al's lask completely trivial for these sentences. In our approach, all test sentences were ambiguous, resulting in a much more diflicult lask. $A$ quantilative comparison belween our model and Jolmson en al.'s is therefore meaningless.

Finally, we are interested in the impact of functional structures on predicting the correct constituent structures. We therefore removed all $i$ structure units from the fragments (hus yickding a TreeDOP model) and compared the results against our version of $\mathrm{LFG}$-DOP (which include the Discardgenerated fragments). We evaluated the parse accuracy on the tree-structures only, using exact match rogethes with the PARSIiVAI measures. We used the same training/test set splits as in the previous experiments and limited the maximum subtree depth again to 4 . The following tables show the results.

\begin{tabular}{lccc}
\hline & Lxact Match & Precision & Recall \\
\hline Tree-DOP & $46.6 \%$ & $88.9 \%$ & $86.7 \%$ \\
LFG-DOP & $50.8 \%$ & $90.3 \%$ & $88.4 \%$ \\
\hline
\end{tabular}

Table 3. C-structure accuracy on the Verbmobil

\footnotetext{
t Although generalized fragments thus seem statistically unimportant for these corpora, they remain important for parsing ungrammatical sentences (which was the original motivation for including them -- see Bod \& Kaptan 1998).
} 


\begin{tabular}{lccc}
\hline & Exact Match & Precision & Recall \\
\hline Tree-DOP & $49.0 \%$ & $93.4 \%$ & $92.1 \%$ \\
LFG-DOP & $53.2 \%$ & $95.8 \%$ & $94.7 \%$ \\
\hline
\end{tabular}

Table 4. C-structure accuracy on the Homecentre

The results indicate that $L F G-D O P$ 's functional structures help to improve the parse accuracy of treestructures. In other words, LFG-DOP outperforms TreeDOP if evaluated on tree-structures only. According to paired $t$-tests the differences in accuracy were statistically significant.

\section{Conclusion}

We have given an empirical assessment of the LFGDOP model introduced by Bod \& Kaplan (1998). We developed a new probability model for LFG-DOP which treats fragments with generalized features as previously unseen events. The experiments showed that our probability model outpertorms Bod \& Kaplan's model on the Verbmobil and Homecentre corpora. Moreover, Bod \& Kaplan's model turned out to be inadequate in dealing with generalized fragments. We also established that the contribution of generalized fragments to the parse accuracy in our model is minimal and statistically insignificant. Finally, we showed that LFG's functional structures contribute to signilicantly higher parse accuracy on tree structures. This suggests that our model may be successfully used to exploit the functional annotations in the Penn Treebank (Marcus et al. 1994), provided that these annotations can be converted into LFG-style functional structures. As future research, we want to test LFG-DOP using log-linear models, as such models maximize the likelihood of the training corpus.

\section{References}

M. van den Berg, R. Bod and R. Scha, 1994. "A Corpus-Based Approach to Scmantic Interpretation", Proceedings Ninth Ansterdam Colloquium, Amsterdam, The Netherlands.

E. Black et al., 1991. "A Procedure for Quantitatively Comparing the Syntactic Coverage of English", Proceedings DARPA Speech and Natural Language Workshop, Pacific Grove, Morgan Kaufmann.

R. Bod, 1993. "Using an Annotated Language Corpus as a Virtual Stochastic Grammar", Proceedings AAAI'93, Washington D.C.

R. Bod, 1998. Beyond Gramnar, CSLI Publications, Cambridge University Press.

R. Bod, 2000. "Parsing with the Shortest Derivation", Proceedings COLING-2000, Saarbrücken, Germany.

R. Bod and R. Kaplan, 1998. "A Probabilistic Corpus-Driven Model for Lexical Functional Analysis", Proceedings COLING-ACL'98, Montreal, Canada.
R. Bonnema, R. Bod and R. Scha, 1997. "A DOP Model for Semantic Interpretation", Proceedings ACL/EACL-97, Madrid, Spain.

J. Chappelier and M. Rajman, 1998. "Extraction stochastique d'arbres d'analyse pour le modèle DOP", Proceedings TALN'98, Paris, France.

B. Cormons, 1999. Analyse et désambiguisation: Une approche à base de corpus (Data-Oriented Parsing) pour les répresentations lexicales fonctionnelles. $\mathrm{PhD}$ thesis, Université de Rennes, France.

I. Good, 1953. "The Population Frequencies of Species and the Estimation of Population Parameters", Biometrika 40, 237-264.

J. Goodman, 1998. Parsing Inside-Out, PhD thesis, Harvard University, Mass.

M. Johnson, S. Geman, S. Canon, Z. Chi and S. Riczler, 1999. "Estimators for Stochastic Unification-Based Grammars", Proceedings $A C L ' 99$, Maryland.

M. Johnson and S. Riezler, 2000. "Exploiting Auxiliary Distributions in Stochastic Unification-Based Grammars", proceedings ANLP-NAACL-2000, Scattle, Washington.

R. Kaplan, and J. Bresnan, 1982. "Lexical-Functional Grammar: A Formal System for Grammatical Representation", in J. Bresnan (ed.), The Mental Representation of Grammatical Relations, The MIT Press, Cambridge, Mass.

M. Marcus, G. Kim, M. Marcinkiewicz, R. MacIntyre, A. Bies, M. Ferguson, K. Katz and B. Schasberger, 1994. "The Penn Treebank: Annotating Predicate Argument Structure". In: ARPA Human Language Technology Workshop, 110-115.

J. Maxwell and R. Kaplan, 1991. "A Method for Disjunctive Constraint Satisfaction", in M. Tomita (cd.), Current Issues in Parsing Technology, Kluwer Academic Publishers.

G. Neumann and D. Flickinger, 1999. "Learning Stochastic Lexicalized Tree Grammars from HPSG", DFKI Technical Report, Saarbrücken, Germany.

H. Ney, S. Martin and F. Wessel, 1997. "Statistical Language Modeling Using Leaving-One-Out", in S. Young \& G. Bloothooft (eds.), Corpus-Based Methods in Language and Speech Processing, Kluwer Academic Publishers.

K. Sima'an, 1995. "An optimized algorithm for Data Oriented Parsing", in R. Mitkov and N. Nicolov (eds.), Recent Advances in Nattral Language Processing 1995, volume 136 of Current Issues in Linguistic Theory. John Benjamins, Amsterdam.

K. Sima'an, 1999. Learning Efficient Disambiguation. PhD thesis, ILLC dissertation series number 1999-02. Utrecht / Amsterdam.

D. Tugwell, 1995. "A Statc-Transition Grammar for DataOriented Parsing", Proceedings European Chapter of the ACL'95, Dublin, Ireland.

A. Way, 1999. "A Hybrid Architecture for Robust MT using LFG-DOP", Journal of Experimental and Theoretical Artificial Intelligence 11 (Special Issue on MemoryBased Language Processing) 